\title{
Different predictors of memory scanning with unidimensional and digit stimuli
}

\author{
ROBERT M. LEVY, DAVID M. GOLDBERG, and JOHN C. SCHMID \\ Indiana State University, Terre Haute, Indiana 47809
}

\begin{abstract}
The processing of digit and unidimensional brightness stimuli was examined in the traditional Sternberg fixed-set paradigm. From a total set size of 10,18 different memory sets were studied, 6 each at memory set sizes of 1,2 , and 3 . Three different variables, which are in general correlated, were used to predict performance: memory set size (M), a measure based on inferred distance relationships among the stimuli constituting the positive and negative sets $(\bar{d})$, and a measure based on the number of inferred "decision boundaries" necessary to separate all memory set items from the items remaining in the set (DB). For the digit stimuli the results were consistent with previous research, and memory set size was the prepotent predictor. For the unidimensional brightnesses, DB was the prepotent predictor, and choice reaction times (CRTs) to both positive and negative probes were a function of the distance of the probe item from the closest member of the complementary set. However, for the digit stimuli this was found only for negative probes; CRTs to positive digit probes were independent of the distance from the closest negative set member.
\end{abstract}

Psychological distance as measured by stimulus similarity has been shown to predict performance in identification tasks in which subjects name stimuli from a set of alternatives (Lockhead, 1970; Monahan \& Lockhead, 1977), as well as in classification tasks in which subjects sort stimuli on the basis of some attribute (Lockhead \& King, 1977). Researchers interested in distance effects have typically employed simple stimuli that vary along one or two perceptual dimensions, such as size, brightness, or linear extent. In direct contrast, highly familiar and complex stimuli, such as digits, letters of the alphabet, words, and so on, have been employed in a similar experimental paradigm, the Sternberg item recognition paradigm (Sternberg, 1975), in which subjects decide whether or not a probe item is a member of a memorized set. In that the Sternberg paradigm bears much in common with both identification and classification tasks, one might reason that distance, which predicts performance in the latter two tasks, should likewise be a factor in predicting performance in the former. The purpose of the present research was to compare the processing of two kinds of stimuli, digits and unidimensional brightnesses, within the framework of the Sternberg paradigm.

Analysis of the two kinds of stimuli leads to some suppositions about their processing. A set of unidimensional stimuli corresponds to a set of digit stimuli in an obvious way: The values form ordered sets; the former

This research was supported, in part, by an Indiana State University Research Foundation grant to the first author. Michael Kubovy and Gregory Lockhead's comments, John Baldasare's assistance with the analysis, and, especially, Jayne VanBramer's assistance in the replication of one of the conditions are gratefully appreciated. Requests for reprints should be sent to Robert M. Levy, Department of Psychology, Indiana State University, Terre Haute, Indiana 47809. have a perceptual order, and the latter have a learned order. It is assumed that the concept of order implies the more general concept of distance. With a set of perceptually ordered unidimensional stimuli, it is reasonable to assume that the distance, $d$, between stimuli affects discriminability, that is, how easy it is to judge two stimuli to be different. Furthermore, differences in discriminability will be assumed to lead to differences in choice reaction time $(C R T)$; if $d(x, y)<d(x, z)$, then CRT to $x$ vs. $y>C R T$ to $x$ vs. $z$.

The distance variable has implications for two major variables that affect processing in the Sternberg (1975) paradigm, the size of the memory set and the items that constitute the memory set. Let $d$ be the distance between a member of either the positive or the negative set and the closest member of the complementary set. For example, assuming equal intervals are reflected in the ordered values, the values of $d$ for the positive set $\{3,7\}$ respective to the negative set $\{0,1,2,4,5,6,8$, 9) are 1 and 1 , as 3 is 1 unit away from the negative elements 2 and 4 , and 7 is 1 unit away from the negative elements 6 and 8. Similarly, the values for $d$ for the negative set $\{0,1,2,4,5,6,8,9\}$ respective to the positive set are $3,2,1,1,2,1,1$, and 2 . The average of these distances of the positive and negative set members is taken as a measure of overall distance, $\overline{\mathrm{d}}$; that is, $\overline{\mathrm{d}}=\Sigma \mathrm{d} / \mathrm{n}$, where $\mathrm{n}$ is the total number of elements. Thus, for the set $\{3,7\}, \bar{d}$ is 1.5 .

When memory set size is manipulated, $\overline{\mathrm{d}}$, averaged over memory sets of the same size, will first decrease as memory set size increases up to one-half the total set size and then increase as memory set size increases to the total set size. In general, for sets of a given size, $\bar{d}$ tends to be larger for grouped elements, such as $\{0,1,2\}$ and $\{3,4,5\}$, than for ungrouped elements, such as $\{0,6,9\}$ and $\{2,5,7\}$. Therefore, if CRT is a 
monotonic function of distance, then the overall CRTs for different positive sets of the same size should decrease as $\bar{d}$ increases. Such a result would be consistent with previous research using digit stimuli (e.g., DeRosa \& Beckwith, 1971; DeRosa \& Morin, 1970; Marcel, 1970), in which "distance" was examined within the context of the organization of the positive set.

The concept of distance, however, becomes functionally relevant only in the context of a decision process involving the discrimination of elements within that space. The concept of a "decision boundary" is thus an essential and corollary concept to distance. Within the context of the Sternberg (1975) paradigm, it is proposed that there are, or may be, decision boundaries between members of the positive set and members of the negative set. For example, with the total set $\{0,1, \ldots, 9\}$, a positive set $\{0\}$ would have one boundary, the positive set $\{3\}$ would have two, and the set $\{3,7\}$ would have four. If decision boundaries represent points of confusability, then CRT should increase as the number of decision boundaries, $\mathrm{DB}$, increases.

But, when different memory sets of different sizes are analyzed for the abstractly defined ordered sets of the kind just described, a basic relationship becomes evident; namely, memory set size (M), overall distance $(\bar{d})$, and the number of decision boundaries (DB) are highly correlated. For example, among the 18 stimulus sets used in the present experiment, the Pearson productmoment correlations were: $\mathrm{r}_{\mathrm{M}, \mathrm{DB}}=.385, \mathrm{r}_{\mathrm{M}, \overline{\mathrm{d}}}=-.511$, and $\mathrm{r}_{\mathrm{DB}}, \overline{\mathrm{d}}=-.857$. As a starting point, therefore, the goal of the present study was, with the use of multipleregression techniques, to determine the roles of these three correlated variables in the processing of two potentially different classes of stimuli, that is, digits and unidimensional brightnesses, in the Sternberg (1975) fixed-set paradigm.

\section{METHOD}

Subjects

Twelve undergraduate students at Indiana State University volunteered to serve as subjects. Six subjects were randomly assigned to the digit and brightness groups. Due to incomplete counterbalancing and high error rates in the brightness group, a replication was run with six additional subjects, and it is these latter data that are reported.

\section{Apparatus}

Stimuli were presented in one channel of a Scientific Prototype Model 800E two-channel tachistoscope. There was a blank white pre- and postexposure field of approximately the same illumination as the stimulus field. A double-throw response lever was situated directly in front of the subject.

\section{Conditions}

There were two types of stimuli, digits and brightnesses. The digit stimuli were the digits $0,1, \ldots, 9$ in pica type typed in the center of $10.2 \times 12.7 \mathrm{~cm}$ white cards for presentation in the tachistoscope. The brightness stimuli were disks, mounted on white cards, of 10 shades of Munsell neutral gray from $\mathrm{N} 2.25$ to $\mathrm{N} 9.00$ in steps of .75 . The diameter of the disks was $1.60 \mathrm{~cm}$. The brightness stimuli were designated $0,1, \ldots, 9$ in order of increasing brightness to correspond to the elements in the digit set.
Table 1

Positive Set Elements, Memory Set Size (M), Average Overall Distance $(\overline{\mathrm{d}})$, Number of Decision Boundaries (DB), and Choice Reaction Times (CRT) for Digit and Brightness Stimuli

\begin{tabular}{rccccc}
\hline & & & & \multicolumn{2}{c}{ Overall CRT } \\
\cline { 3 - 6 } Set & M & $\bar{d}$ & DB & Digit & Brightness \\
\hline 0 & 1 & 4.6 & 1 & .393 & .833 \\
2 & 1 & 3.2 & 2 & .414 & 1.000 \\
4 & 1 & 2.6 & 2 & .388 & .880 \\
5 & 1 & 2.6 & 2 & .393 & .915 \\
7 & 1 & 3.2 & 2 & .387 & .894 \\
9 & 1 & 4.6 & 1 & .407 & .732 \\
0,1 & 2 & 3.9 & 1 & .416 & .956 \\
0,9 & 2 & 2.2 & 2 & .424 & .841 \\
1,8 & 2 & 1.6 & 4 & .455 & .972 \\
3,6 & 2 & 1.6 & 4 & .443 & 1.395 \\
4,5 & 2 & 2.2 & 2 & .441 & 1.078 \\
8,9 & 2 & 3.9 & 1 & .434 & .794 \\
$0,1,2$ & 3 & 3.4 & 1 & .442 & .740 \\
$0,4,9$ & 3 & 1.3 & 4 & .405 & 1.170 \\
$0,6,9$ & 3 & 1.4 & 4 & .445 & 1.202 \\
$2,5,7$ & 3 & 1.2 & 6 & .454 & 1.709 \\
$3,4,5$ & 3 & 2.0 & 2 & .479 & 1.066 \\
$7,8,9$ & 3 & 3.4 & 1 & .460 & .877 \\
\hline
\end{tabular}

The positive sets were selected systematically rather than randomly in order to facilitate investigation of distance and decision boundary effects. The different memory sets, the values of overall distance $(\bar{d})$, and the number of decision boundaries (DB) are shown on the left side of Table 1.

\section{Procedure}

On each of 6 consecutive days, the subject participated in three separate sessions of 74 trials each (10 practice and 64 test trials for digits and first replication for brightnesses) or 78 trials each (24 practice and 54 test trials for second, reported replication of brightnesses). The order in which memory sets occurred was counterbalanced over the 6 days, and the sessions were counterbalanced within each day.

During instructions, the concepts "positive set" and "negative set" were defined, and the subject was instructed to respond to positive stimuli by moving the response lever one way and to negative stimuli by moving it the other way. Accuracy of response was emphasized over speed, and accuracy feedback was given to the subject after each trial.

On approximately $50 \%$ of the trials, stimuli were from the positive set, and stimuli within the positive and negative sets occurred equally often. A trial began with a 200 -msec warning tone followed by a 300 -msec quiet interval before stimulus field onset. The subject's response switched the stimulus field to the postexposure field and stopped the clock.

\section{RESULTS}

In order to determine the relative weightings of memory set size, distance, and decision boundaries as predictors of overall CRT performance, multipleregression equations were obtained. The data, also shown in Table 1 , were the 18 overall correct ${ }^{1}$ CRTs averaged across the six subjects in each condition. The two regression equations, with predictor variables entered in order of contribution, were digits: $\mathrm{CRT}=.389+$ $.025 \mathrm{M}-.003 \overline{\mathrm{d}}-.002 \mathrm{DB}[\mathrm{F}(3,14)=6.79, \mathrm{p}<.01]$, and brightness: $\mathrm{CRT}=.423+.166 \mathrm{DB}+.042 \mathrm{M}+$ $.040 \bar{d}[F(3,14)=18.5, p<.01]$. For digits, the coefficients of determination were $.590, .591$, and .592 , 
with the entry of $M, \bar{d}$, and $D B$ into the regression equation, respectively, and for brightnesses the coefficients of determination were $.780, .790$, and .797 , with the entry of $\mathrm{DB}, \mathrm{M}$, and $\overline{\mathrm{d}}$, respectively.

The results were straightforward. For the digit stimuli, memory set size was the best predictor of performance in the Sternberg (1975) paradigm. Since DB and $\bar{d}$ did not contribute significantly to predictability, the traditional regression equations for $\mathrm{CRT}$ vs. $\mathrm{M}$ were examined: positive $\mathrm{CRT}=.344+.033 \mathrm{M}[\mathrm{F}(1,16)=50.8$, $\mathrm{p}<.01] ;$ negative $\mathrm{CRT}=.397+.026 \mathrm{M}[\mathrm{F}(1,16)=36.5$, $\mathrm{p}<.01]$. The positive CRT vs. memory set size function for digits showed a greater increase in CRT from $M_{1}$ to $M_{2}$ (45 msec/item) than from $M_{2}$ to $M_{3}(20 \mathrm{msec} / \mathrm{item})$, which might account for the relatively small coefficients of determination in the multiple-regression equation for digits. The nonlinearity for digits may, as Marcel (1977) and Nickerson (1972) have discussed in some detail, reflect that sets of Size 1 might be processed differently. From $M_{2}$ to $M_{3}$ the positive and negative functions for digits were parallel, with slopes of $20 \mathrm{msec} / \mathrm{item}$.

The corresponding meaningful analysis for the brightness stimuli is the CRT vs. DB functions: positive $\mathrm{CRT}=.690+.131 \mathrm{DB}[\mathrm{F}(1,16)=27.8, \mathrm{p}<.01] ;$ negative $\mathrm{CRT}=.604+.170 \mathrm{DB}[\mathrm{F}(1,16)=72.8, \mathrm{p}<.01]$. Although neither $\overline{\mathrm{d}}$ nor $\mathrm{M}$ contributed significantly to the prediction of positive CRTs, for future reference it is noted that the inclusion of $\mathrm{M}$ with $\mathrm{DB}$ in the prediction equation for negative CRTs did. The intercept for negative CRT is less than the intercept for positive CRT, but the functions intersect. Since DB and $\bar{d}$ are negatively correlated, the memory sets with fewer decision boundaries were those that contained the greater interset distances. Therefore, the negative CRTs for small DB sets (also the small memory sets) were weighted more heavily by short CRTs.

\section{Interset Distance Effects}

The positive and negative CRTs as a function of interset distance, $d$, for each set size are shown in Figure 1. The CRTs were averaged to the stimuli at each distance within a condition and then averaged across different positive sets and, consequently, different numbers of decision boundary conditions at the given memory set size.

Negative CRTs for both the digit stimuli $[F(6,30)=$ $5.23, \mathrm{p}<.001]$ and the brightness stimuli $[\mathrm{F}(6,30)=$ $9.6, \mathrm{p}<.001$ ] decreased as distance increased until a distance of three or four was reached. Distance and memory set size interacted for both the digit stimuli $[F(12,60)=3.38, p<.001]$ and the brightness stimuli $[F(12,60)=3.6, p<.001]$; however, the forms of the interaction were different. For the digit stimuli, a major component of the interaction was the flatness of the

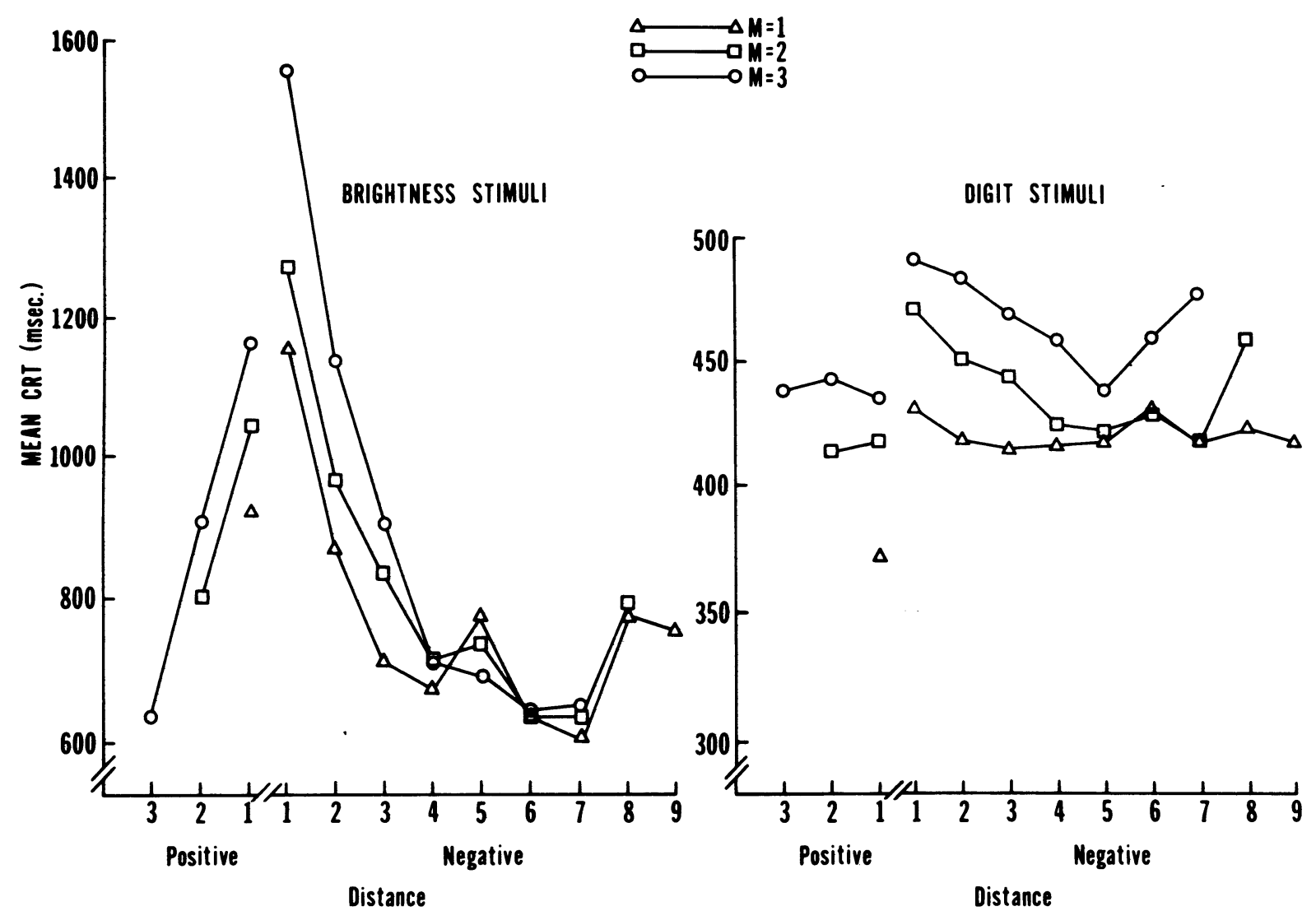

Figure 1. Choice reaction time to positive and negative stimuli as a function of the distance from the closest member of the complementary set and memory set size. 
distance function for $M_{1}$ compared with the distance functions for $M_{2}$ and $M_{3}$. Two important results were: (1) CRTs were longer for $M_{3}$ stimuli at all distances than for $M_{2}$ stimuli, that is, there was always an effect of memory set size; and (2) the relative flatness of the distance function for $\mathbf{M}_{1}$, which is consistent with Marcel (1977), supports the interpretation of different processing for that condition.

For the brightness stimuli, the positive correlation between set size and average number of decision boundaries will account for the apparent set size effect. The independence between set size and CRT at distances of four or greater can also be attributed to this correlation, since memory sets for which there were interset distances of four and greater in the present experiment were those with one decision boundary. The independence of distance and CRT at the larger distances probably reflects the subjects' adopting a more efficient strategy than processing the stimuli with regard to the decision boundary. A modification of Atkinson and Juola's (1974) suggestion that the memory set need not be searched for items either low or high in familiarity might serve as a basis for interpreting the result: Especially in the fixed-set procedure, subjects might establish a category, "easy negative decision," and scan for that category before they undertake a more systematic search of the categories defined by the decision boundaries per se, which involves difficult discriminations.

Positive CRTs decreased with distance for the brightness stimuli, but positive CRTs were independent of distance for digit stimuli. If distance is functional only with regard to points of confusability, then distance should not operate where confusability is minimal or nonexistent. Digit stimuli are very familiar and highly discriminable, representing stimuli that have minimal perceptual confusability. However, the highly learned names to the stimuli might be a basis for confusion of a different kind. To speculate, if for digit stimuli, subjects first search the positive set and a positive probe is presented, a match will be made, and there will be no confusability at the time of decision. But suppose a negative probe is presented but the positive set is searched. The negative stimulus might, based on associative relationships, "arouse" a tendency to respond to a positive set member in proportion to associative strength, which would correspond to distance. At the time of decision, therefore, there would be confusion and consequent distance effects for negative CRTs.

\section{DISCUSSION}

Brightness and digit stimuli surely represent different classes of stimuli. The brightness stimuli were the ultimate in simplicity: unidimensional with a natural order, but, from the subjects' viewpoint, unfamiliar. The number of what we have called "decision boundaries" was the primary determinant of overall performance and accounted for memory set size effects. There were interset distance effects for both positive and negative stimuli, consistent with a confusion model of processing. With the unidimensional stimuli, defined by their "physical" and thus their perceptual properties, it is relatively easy to develop a simple model for the distance relationships and "decision boundaries" implied in spatial relationships. In the Sternberg (1975) paradigm, distance is now defined in terms of sets of stimuli. The stimulus-response mapping, that is, the task, defines the set of functional distance relationships and the correlated decision boundaries.

The digit stimuli were complex visual forms of unspecified dimensionality, but very familiar, with a learned, or derived, order. Although there were interset distance effects for negative digit stimuli associated with their order, memory set size per se was the primary determinant of performance, and, it would seem, processing was with regard to the memory set. A corresponding generalization of stimuli to which the digit processing. applies is not as straightforward as it is for the brightnesses. Digits are in a sense a relatively unique set of stimuli, since learned order is their defining characteristic and the basis of their meaning. The general property would seem to be sets of stimuli defined by meaning in the semantic sense, relationships with a corresponding "semantic space."

Finally, the purpose of the present research was not theory testing (e.g., Monsell, 1978) per se. Rather, it was to determine how two different classes of stimuli are processed. Clearly, the juxtaposed digits and brightnesses led to different processing. The problem remains to determine what variables transform one kind of processing to the other.

\section{REFERENCES}

Atrinson, R. C., \& Juola, J. F. Search and decision processes in recognition memory. In D. H. Krantz, R. C. Atkinson, R. D. Luce, \& P. Suppes (Eds.), Contemporary developments in mathematical psychology. San Francisco: Freeman, 1974.

DeRosa, D., \& Beckwith, M. Retrieval of information from organized memory sets. Psychonomic Science, 1971, 23, 177-179.

DeRosa, D., \& Morin, R. Recognition reaction time for digits in consecutive and nonconsecutive memorized sets. Journal of Experimental Psychology, 1970, 83, 472-479.

LockHEAD, G. Identification and the form of multidimensional discrimination space. Journal of Experimental Psychology, 1970, 85, 1-10.

LockHE AD, G., \& KING, M. Classifying integral stimuli. Journal of Experimental Psychology: Human Perception and Performance, 1977, 3, 436-443.

MARCEL, A. Some constraints on sequential and parallel processing and the limits of attention. Acta Psychologica, 1970, 33, 77-92.

MarCeL, A. J. Negative set effects in character classification: A response retrieval view of reaction time. Quarterly Journal of Experimental Psychology, 1977, 29, 31-48.

Monahan, J., \& Lockhe AD, G. Identification of integral stimuli. Journal of Experimental Psychology: General, 1977, 106, 94-110.

Monsell, S. Recency, immediate recognition memory, and reaction time. Cognitive Psychology, 1978, 10, 465-501.

Nicke RSON, R. Binary-classification reaction time: A review of some studies of human information processing capabilities. Psychonomic Monograph Supplements, 1972, 4, 274-318.

Sternberg, S. Memory scanning: New findings and current controversies. Quarterly Journal of Experimental Psychology, 1975, 27, 1-32.

\section{NOTE}

1. For digit stimuli, there were errors on approximately $.7 \%$ of the trials. For the brightness stimuli, there were errors on approximately $8.4 \%$ of the trials, and the number of errors was a decreasing function of the distance.

(Received for publication March 8, 1980.) 